\title{
Laparoscopic Splenectomy with Preoperative Embolization for Splenic Metastasis of an Endometrial Adenocarcinoma
}

\author{
Louis Smits ${ }^{1,2 *}$, Aude Schillaci ${ }^{2}$, Sorin Cimpean ${ }^{3}$, Etienne Veys ${ }^{2}$ \\ ${ }^{1}$ Assistant UCL, General Surgery, Notre Dame de Grâce Clinic (CNDG), Gosselies, Belgium \\ ${ }^{2}$ Notre Dame de Grâce Clinic, Gosselies, Belgium \\ ${ }^{3}$ Saint Pierre University Hospital, Brussels, Belgium
}

*Corresponding Author: Louis Smits, Assistant UCL, General Surgery, Notre Dame de Grâce Clinic (CNDG), Gosselies, Belgium, Email : louis.smits@hotmail.be

\begin{abstract}
Introduction: Given the anatomical specificities of the spleen and its particular microenvironment, splenic metastases of an adenocarcinoma are exceptional.

Case presentation: We present the case of a 74-year-old patient with a unique splenic metastasis following endometrial cancer diagnosed and treated five months previously. We performed a laparoscopic splenectomy with preoperative embolization.

Discussion: Most splenic metastases are found in multi-metastatic cancer settings with poor prognosis and do not involve systematic surgical management. In rare cases single spleen metastases have been described, which can justify a total splenectomy with adjuvant chemotherapy. If preoperative management involves the administration of specific vaccines to the patient, education of the latter in the face of post-operative infectious risks is also essential.
\end{abstract}

Conclusion: The treatment of choice for single splenic metastasis is splenectomy followed by chemotherapy. Splenectomy may be associated with preoperative splenic embolization to reduce the risk of intraoperative hemorrhage and the risk of conversion.

Keywords: Splenic metastases, spleen, endometrial, splenectomy, embolization

\section{INTRODUCTION}

Splenic metastases of adenocarcinoma are rare. Those that follow an endometrial cancer are exceptional. Less than 20 cases have been reported in the literature. The exceptional character of this type of metastasis would be related to the anatomical specificities of the spleen and its particular microenvironment.

We report the case of a 74-year-old patient who, after being treated for endometrioid adenocarcinoma pT1a in August 2018, presented, at a distance, a unique metastasis of splenic localization. We performed a preoperative splenic embolization, followed by a laparoscopic total splenectomy with adjuvant chemotherapy.

\section{Case Presentation}

A 74-year-old patient, with no particular history, consulted her gynecologist in early July 2018 following repeated episodes of metrorrhagia. An ultrasound revealed a $16 \mathrm{~mm}$ intrauterine polyp that was resected. The pathology of the patch revealed endometrioid grade 2 adenocarcinoma, estrogen and progesterone,$+ \mathrm{P} 16+$, CEAreceptors, and no microsatellite instability.

In mid-July 2018, an extension assessment was performed with a negative chest radiograph, a negative whole-body bone scintigraphy and an abdominal ultrasound of the liver, bile ducts, right kidney and also negative pancreas. An MRI of the pelvis revealed, meanwhile, a small mass at the level of the endometrium with homogeneous enhancement located in the right lateral not infiltrating the myometrium.

A complete laparoscopic radical hysterectomy was performed on 12 August. The anatomopathological exam of the resected part revealed a well-differentiated endometrioid adenocarcinoma (Grade 1) infiltrating 10\% of the thickness of the myometrium, no angio-lymphatic or vascular penetration, and appendages and a negative neck. Given the stage pT1a, it was 
decided in multidisciplinary consultation to follow the evolution of the patient to one year without further treatment.

In early February 2019, the patient presented herself in the emergency department for epigastric pain and left hypochondrium. An abdominal CT scan revealed a bilobed splenic lesion on the upper inner side of the spleen with moderately thick walls, a hypodense center, and an enhancing periphery with microbulled contours of indeterminate origin. (Fig 1) A Pet Scan confirmed that only the splenic focus enhanced, and showed a bilobal mass of necrotic appearance. The CA 125 was increased to $43 \mathrm{KU}$ / L. A radiologic embolization of the spleen was performed and the splenectomy was performed on March 21st by laparoscopy. (Fig2) The immediate operative follow-up was simple and the patient returned home five days after the procedure.

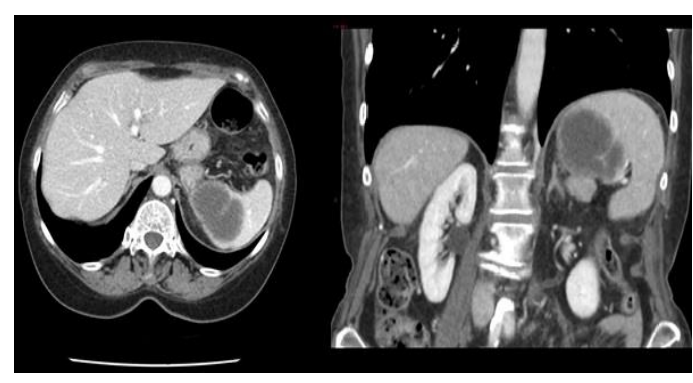

Figure1. Abdominal CT Scan-Bilobed splenic lesion on the upper inner side of the spleen with moderately thick walls, a hypodense center, and an enhancing periphery with microbulled and irregular contours.

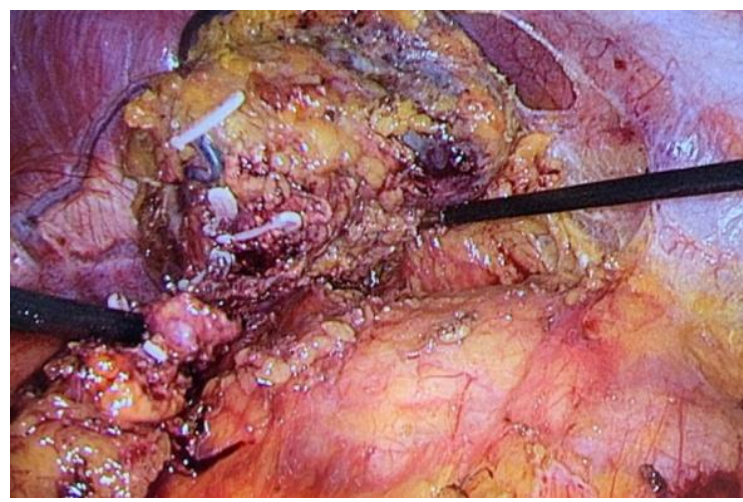

Figure2. Peri-operative image showing the splenic hilum dissection

The anatomopathologic exam of the part revealed a metastasis adenocarcinoma moderately differentiated of the endometrium of $6 \mathrm{~cm}$ long axis with infiltration of the splenic hile. The immunohistochemical profile, for its part, was compatible with an endometrial origin.

Adjuvant treatment of chemotherapy Carboplatin Taxole type was performed later.

\section{DISCUSSION}

The spleen is not a frequent site of metastases. There are two types of splenic metastases: intraparenchymal tumors from hematogenous tumor emboli and capsular metastases found during peritoneal dissemination of advance cancer (ovaries, colon). (1)

Most of the splenic metastases are found in multimetastasized cancer contexts with poor prognosis (2) and do not usually imply systematic surgical management. Isolated splenic metastases, on the other hand, are exceptional lesions (2) (3) and may justify total splenectomy with adjuvant chemotherapy (1).

Cysts and splenic tumors are rare. Generally asymptomatic, they are most often discovered by chance (4). The context, the patient's personal antecedents (cancer, trauma) and the possible symptomatology (fever, left hypochondrial pain) help to guide the diagnosis. In case of doubt, a spleen biopsy is still possible, but this involves a high risk of haemorrhage and tumor dissemination and is therefore generally not recommended (4).

When isolated, splenic metastases are predominantly asymptomatic (3) (1) (60\% of cases (2)). Some symptoms may attract attention, abdominal pain in left hypochondrium, epigastric, cases of splenomegaly associated with thrombocytopenia, fever, weight loss, fatigue (2) (4). Rarely, cases of splenic rupture are described, but they are considered as absolute emergencies. In the majority of cases, splenic metastases are detected during oncology followups by means of an abdominal CT scan or ultrasound scan (5). Some cases of splenic metastases have been demonstrated on the basis of an increase in tumor markers (CEA, PSA, CA125) (6) (5) and subsequently to imaging. As for these markers, their quantity decreases after splenectomy in the cases reported. This was also the case with our patient, where we noticed a standardization of CA125.

Splenic metastases can be discovered synchronously or metachronously (7) (2). In our case, the absence of splenic imaging and symptomatology during the discovery of the primitive did not allow us to conclude whether this metastasis was already present or not at the time of the initial diagnosis. Splenic metastases can occur in three ways: macronodular, as in our case, micronodular or diffuse (1). 
Isolated metastatic splenic lesions are rare and are mainly of ovarian, pulmonary, mammary, melanoma or colorectal origin (3) (1) (6) (8).

Unique spleen metastases of endometrial origin are exceptional. Less than 20 cases have been described in the literature (1). Usually, endometrial cancers metastasize after invasion of the myometrium into the lymph nodes or hematogenously usually to the liver, lungs or bones (1). Spleen metastases of endometrial cancer are usually solitary, localized in the splenic parenchyma. This dissemination is by hematogenous tumor microemboli to the spleen (1). The literature reports that there is a latency period of several months (2) (ranging from 11 to 120 months (1)) before the discovery of splenic metastasis of endometrial cancer. In our case, splenic metastasis was only demonstrated after an interval of 5 to 6 months following the onset of pain in the left hypochondrium.

The rarity of splenic metastases is based on anatomical mechanical factors such as a sharpy angle formed by the splenic artery that makes it difficult to pass tumoral emboli into the spleen, the rhythmic contractions of the splenic capsule prevent tumor emboli deposition, and the absence of afferent lymphatic vessels. Another hypothesis has been proposed, the splenic microenvironment not being favorable to the growth of metastases. Indeed, the spleen has many lymphoid tissues that makes the medium unsuccessful for the deposition and development of tumor emboli in the splenic parenchyma. (2) (3) (1) (6) (8)

\section{Pre-Splenectomy Assessment}

Prevention at the time of patient preparation for splenectomy begins with vaccination targeting the encapsulated organisms (Streptotoccus Pneumoniae, Neisseriae Meningitis and Haemophilus Influenzae type B) (9) (10). Vaccination is recommended at least two weeks before any scheduled splenectomy or two weeks after an emergency splenectomy. Note that the vaccine response is lower two weeks after splenectomy. (10) Moreover, given the risk of pneumococcal superinfection in case of influenza, an annual vaccination against influenza is also recommended. (10)

\section{Splenic Embolization}

Embolization would reduce the risk of hemorrhage bleeding (11) (35 ml with embolization versus 350 $\mathrm{ml}$ without embolization) (12), but also reduce the volume of the spleen intraoperatively in case of splenomegaly. Embolization would also reduce the operative time and the risk of conversion in case of perioperative bleeding (11). Splenic postembolization complications can reach about 5\% (9) and are mainly related to post-embolization syndromes (13), accompanied by pain, fever and/or left pleural effusion related to splenic infarction. Cases of coil migration in peri-splenic organs have also been described with risks of pancreatitis. (9) (11) (13)

\section{Laparoscopic Splenectomy}

Delaitre et al. is the first to report a case of laparoscopic splenectomy in 1991 (14) (15). Laparoscopy reduces hospitalization time, postoperative pain and morbidity and has a considerable aesthetic advantage. Laparoscopy is the gold standard for splenectomy, even in cases of major splenomegaly. We chose the technique of splenic suspension, or " Hanging spleen technique " (14). This is a lateral approach in right lateral decubitus (16). This suspension technique gives good access to the spleen. This is suspended from the diaphragm by the phrenosplenic ligament, which gives easy access under visual control to the posterior surface of the spleen and access to the splenic hilum (14) (17) and the tail of the pancreas which is found attracted away from the hile by gravity. As for the stomach, liver and colon, they are attracted to the bottom of the operative field by their own weight (Fig 2)

This technique combined with previous splenic embolization facilitated access to the spleen and allowed us to control the artery and splenic vein at best despite the unexpected inflammatory fibrotic medium found in the splenic hilum (1). The choice of the laparoscopic approach is usually based on the surgeon's experience.

\section{Post-Splenectomy Complications}

Infectious complications are the most important medical complications post-splenectomy and are the most dangerous. Serious general infections can occur in 3\% to 5\% (9) of long-term cases in any splenectomized patient. Subphrenic abscess cases postoperatively are also described in 5\% of cases (9).

The first two to three years post-splenectomy are the most at risk from an infectious point of view. Some authors even offer antibiotic prophylaxis up to two years post-splenectomy (10). Generally, children are at higher risk of infection (10), as are patients with splenectomies following 
haematological malignancy, lymphoma or thalassemia.

Thromboembolic complications are also common in the porto-mesenteric system as well as in the vena cava system, which carries a risk of pulmonary embolism (18).

The incidence of portal vein thrombosis ranges from $1.6 \%$ to $15 \%$ (10) with a median time to onset of 8 to 12 days (10). The risk of thrombosis is greater in cases of cirrhosis or myeloproliferative disorder (9).

Finally, the perioperative risk of caudal pancreatic injury with pancreatic fistula varies from $0.5 \%$ (9) to $3 \%$ (19) according to the authors.

Given postoperative thrombocytosis and thrombotic risk, the patient was put on aspirin (Asaflow $80 \mathrm{mg}$ once daily).

\section{CONClusion}

The discovery of an unknown splenic mass in a patient with a history of neoplasia should be considered potentially malignant even if the primary tumor has been successfully treated years earlier. If implantation of cancer cells into the spleen occurs, detectable and symptomatic metastases are rare. The final diagnosis can only be made after anatomopathological analysis.

The treatment of choice for splenic metastases is splenectomy followed by adjuvant chemotherapy, which may be associated with preoperative splenic embolization to reduce the risk of intraoperative hemorrhage and the risk of conversion.

\section{REFERENCES}

[1] Arif A, Abideen Z.U, Zia N, et al. Metastatic involvement of the Spleen by endometrial adenocarcioma; a rare asylum for a common malgnancy: a case report. BMC Research Notes. [En ligne] BMC Research Notes, 2013. [Citation : 10 May 2019.] http://www.biomedcentral.com /1756-0500/6/476.

[2] Splenic Metastases. Clinicopathologic Presentation, Differential Diagnosis, and Pathogenesis. Compérat E, Bardier-Dupas A, Camparo P, et al. Arch Pathol Lab Med, June 2007, Vol. 131. pp.965-969.

[3] Metastatic Tumors to the Spleen. A 25-Year Clinicopathologic Study. Lam K.Y, Tang V. Arch Pathol Lab Med, April 2000, Vol. 124. pp.526-530.
[4] Alkofer B, Lepennec V, Chiche L. Kystes et tumeurs spléniques: diagnostic et prise en charge. Journal de Chirurgie. Elsevier Masson , 2005, Vol. 142, 1.

[5] Primary Tumors of the Spleen. Fotiadis C, Georgopoulos I, Stoidis C, et al. 2, s.l. : International Journal of Biomedical Science, 2009, Vol. 5.

[6] Why is Isolated Spleen Metastasis a Rare Entity. Genç V, Akbari M, Karaca A. S, et al. 4, pp.452-453, s.l. : Turk Journal Gastroenterol., 2010, Vol. 21.

[7] Splenic Metastases - Not a Frequent Problem, but an Underestimate Location of Metastases: Epidemiology and Course. Sauer J, Sobolewski K, Dommisch K. 135, pp.667-671, s.l. : J Cancer Res Clin Oncol, 2009.

[8] Giovagnoni A, Giorgi C, Goteri G. Tumours of the Spleen. [International Cancer Imaging Society] s.l.: International Cancer Imaging Society, 2005. DOI:10.1102/1470-7330.2005. 0002.

[9] La splénectomie par laparoscopie: indications, principes, résultats. Borie F, Philippe $\mathbf{C}$. Journal de Chirurgie, s.l. : Elsevier Masson, 2009, Vol. 146, pp.336-346. DOI:10.1016/ j.jchir.2009.08.030.

[10] Complications médicales des splénectomies. Buzelé R, Barbier L, Sauvanet A, et al. Journal de Chirurgie Viscérale, s.l. : Elsevier Masson, 2016, pp.289-299, Vol. 153. DOI.org/10.1016/ j.jviscsurg.2016.04.013.

[11] Wu Z, Zhou J, Pankaj P, et al. Comparative treatment and literature review for laparoscopic splenectomy alone versus preoperative splenic artery embolization splenectomy. [Surgical Endoscopy] s.1. : Springer, 2012. DOI 10.1007/s 00464-012-2270-z.

[12] Concommitant Intraoperative Splenic Artery Embolization and Laparoscopic Splenectomy versus Laparoscopic Splenectomy: Comparaison of Treatment Outcome. Naoum JJ, Siberfein EJ, Zhou W, et al. Am J Surg, 2007, Vol. 193, pp.713-718.

[13] An overview of Splenic Embolization. Ahuja C, Farsad K, Chadha M. 205, pp.720-725, s.1. : AJR , 2015. DOI: 10.2214/AJR.15.14637.

[14] Laparoscopic Splenectomy. 16 Years Since Delaitre With Review of Current Literature. Sharma D, Shukla V. 3, s.l. : Surg Laparosc Endosc Percutan Tech, 2009, Vol. 19. http://www.surgical-laparoscopy.com.

[15] Splenectomyby the Laparoscopic approach. Report of a case. . Delaitre B, Maignien B. s.l. : Presse Medicale, 1991, Vol. 20, p.2263. PubMed:PMID:1838167. 
[16] Laparoscopic Splenectomy: Perioperative Management, Surgical Technique, and Results. Fisichella P.M, Wong Y.M, Pappas S.G, et al. 18, pp.404-410, s.1. : J Gastrointest Surg, 2014. DOI: $10.1007 / \mathrm{s} 11605-013-2366-3$.

[17] Munoz-Bongrand N, Sarfati E. Chirurgie des rates pathologiques. Techniques chirurgicales Appareil digestif. EMC Elsevier Masson, 2009, Vol. 40, 753.
[18] Les complications à moyen et long terme de la splénectomie. S., Benoist. 125, pp.317-324, s.1. : Editions médicales et scientifiques Elsevier SAS, 2000. S0003394400002017/SSU.

[19] Management of Postoperative Complications Following Splenectomy. Yikun Q, Shiyan R, Chunmin L, et al. 98, pp.55-60, s.1. : Int Surg, 2013.

Citation: Louis Smits, Aude Schillaci, Sorin Cimpean, Etienne Veys, Laparoscopic Splenectomy with Preoperative Embolization for Splenic Metastasis of an Endometrial Adenocarcinoma. ARC Journal of Clinical Case Reports. 2019; 5(3): 5-9. doi:dx.doi.org/10.20431/2455-9806.0503002.

Copyright: (C) 2019 Authors. This is an open-access article distributed under the terms of the Creative Commons Attribution License, which permits unrestricted use, distribution, and reproduction in any medium, provided the original author and source are credited. 